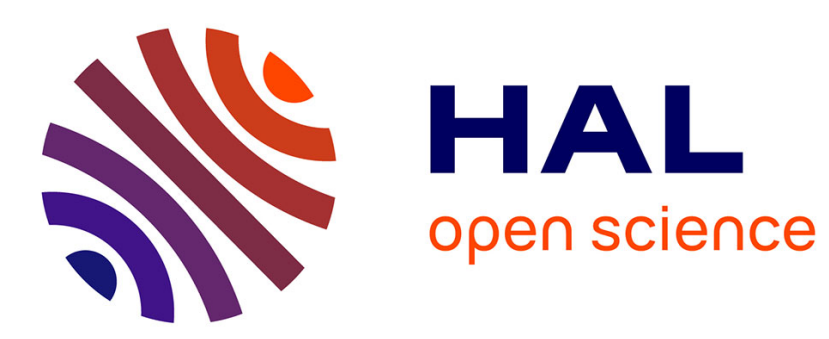

\title{
Study of RCS of complex target: Experimental measurements and Gaussian beam summation method
}

Helmi Ghanmi, Ali Khenchaf, Papa Ousmane Leye, Philippe Pouliguen

\section{To cite this version:}

Helmi Ghanmi, Ali Khenchaf, Papa Ousmane Leye, Philippe Pouliguen. Study of RCS of complex target: Experimental measurements and Gaussian beam summation method. IEEE Conference on Antenna Measurements \& Applications 2017, (CAMA 2017), Dec 2017, Tsukuba, Japan. pp.196-199, 10.1109/CAMA.2017.8273399 . hal-01702322

HAL Id: hal-01702322

https://hal-ensta-bretagne.archives-ouvertes.fr/hal-01702322

Submitted on 20 Jan 2020

HAL is a multi-disciplinary open access archive for the deposit and dissemination of scientific research documents, whether they are published or not. The documents may come from teaching and research institutions in France or abroad, or from public or private research centers.
L'archive ouverte pluridisciplinaire HAL, est destinée au dépôt et à la diffusion de documents scientifiques de niveau recherche, publiés ou non, émanant des établissements d'enseignement et de recherche français ou étrangers, des laboratoires publics ou privés. 


\section{Study of RCS of Complex Target: Experimental Measurements and Gaussian Beam Summation Method}

\author{
H. Ghanmi, A. Khenchaf, P.O.Leye \\ Lab-STICC UMR CNRS 6285, ENSTA Bretagne \\ 29806, Brest, France \\ \{Helmi.ghanmi,Ali.khenchaf@ensta-bretagne.fr\}
}

\author{
P. Pouliguen \\ French General Directorate for Armament (DGA) \\ 75509, Paris, France \\ Philippe.Pouliguen@intradef.gouv.fr
}

\begin{abstract}
This paper presents a numerical and experimental study of RCS of canonical and complex targets using Gaussian Beam Summation (GBS) method. The purposed GBS method has several advantages over ray method, mainly on caustic problem. To test and evaluate the performance of the chosen method, we start the analysis of the RCS using GBS, the asymptotic models Physical Optic (PO), Geometrical Theory of Diffraction (GTD) and the rigorous Method of Moment (MoM). Then, we show the experimental validation of the numerical results using well calibrate measurements of radar targets. These experimental measurements have been carried out in our anechoic chamber (at ENSTA Bretagne). The numerical and experimental results of the RCS are studied and given as a function of various parameters: polarization type, target size, Gaussian beams number and Gaussian beams width.
\end{abstract}

Keywords-Radar Target; Radar Cross Section (RCS); Gaussian Beam Summation (GBS); Physical Optic (PO), Method of Moment (MoM); Geometrical Theory of Diffraction (GTD)

\section{INTRODUCTION}

The simulation of the electromagnetic scattering and the RCS of a canonical or a complex target are used in the implementation of radar target detection. Each simulation requires the implementation of numerical resolution of the differential equations relative to the behavior of the wave. In the radar frequency domain and in the case of the scattering from an object (with large size), both asymptotic and rigorous methods have been developed. The asymptotic method (PO, TGD, TUD) reduce the operation number of solving of high frequency equations as for large objects $[1,2,7]$. The rigorous methods such as MoM are based on an integral formulation, and they are served to validate the new asymptotic approach. The asymptotic method using the hypothesis of locally plane wave and high frequency approximation are based on the principle of rays. The application of the type of these methods in a complex propagation scenario is often limited by the transaction between highlighted and shadowed region and the caustic problem (except the PO method). To overcome this problem, we have chosen a method which is called Gaussian Beam Summation (GBS) [3, 4, 5, 6] [8]. Then, we have applied the GBS method to model the variation of the RCS section of a canonical object which can be extended to the case of a complex scenario. In addition, the main reason for choosing the method is justified by the fact that the GBS eliminate several limitation of the ray asymptotic approach, in particular the difficulties connected with evaluating the wave field in singular regions.
The main goal of this work is to investigate the different mechanisms of electromagnetic wave scattering at canonical and complex objects using GBS method and validate the numerical simulation results by experimental measurements.

\section{FORMULATION OF GBS METHOD}

V. Cerveny [5, 8] and M.M. Popov [6] have developed a new approach for calculation of wave fields in high frequency approximation. This method named Gaussian Beam approach. The physical principle of Gaussian Beam approach consists in a compatible step assembly. In fact, consider an electromagnetic wave propagating in a homogeneous and isotropic medium which is being excited by a point source. In the GBS method, the final field in an observation point results from a fan of rays distributed in his vicinity. For each ray we derive a Gaussian beam propagating along the ray. Then we sum the contribution of each Gaussian beam to the receiver over all rays from the fan [9]. Suppose that the point source is situated at the origin and some wave process is described by the Helmholtz equation with a propagation velocity $v$. We solve the Helmholtz equation in the vicinity of rays. For any selected ray $\Omega$, we shall introduce a ray-centered coordinate system, $q_{1}, q_{2}$ and s connected with $\Omega$ (see Fig. 1) where s corresponds to arc length (curvilinear abscissa) along the ray. A solitary Gaussian beam as a localized asymptotic solution to the Helmholtz Equation calculated at the receiver point, with local coordinates $s, q_{1}, q_{2}$ inside asymptotically small neighborhood of the central ray is given by [6].

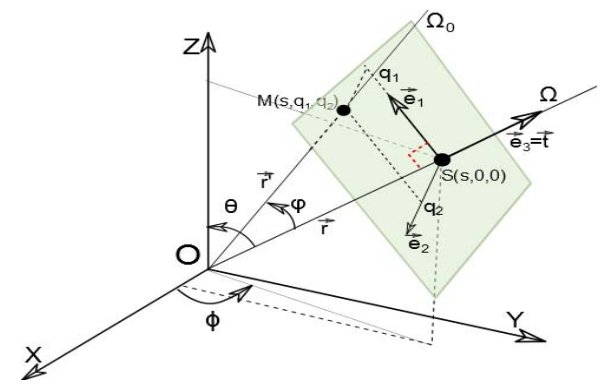

Fig. 1. Ray-centered coordinates $\mathrm{q}_{1}, \mathrm{q}_{2}$, and $\mathrm{s}$ of point $\mathrm{M}$ situated in the vicinity of ray $\Omega$. Point $M$ is situated in plane $\Sigma_{\perp}$ perpendicular and crossing $\Omega$ at point $\mathrm{S}$. The origin $\mathrm{O}$ is at $\mathrm{s}=\mathrm{s}_{0}$.

$$
\left\{\begin{array}{l}
u\left(s, q_{1}, q_{2}, t\right)=\left(\frac{v}{\operatorname{det}[Q]}\right)^{\frac{1}{2}} \cdot \exp \left(-j \omega\left[t-\tau(s)-\frac{1}{2}\left(q^{T} \cdot P \cdot Q^{-1} \cdot q\right)\right]\right) \\
\tau(s)=\int_{s_{0}}^{s} \frac{d s}{v}
\end{array}\right.
$$


Where $\tau(s)$ is the travel time from the source along the selected ray, $v$ is the propagation velocity, $q^{T}$ represents the transpose of the vector, the quantities $Q$ and $P$ are $2 \times 2$ matrix called "dynamic quantites" satisfying the system ODE (2) in variations, called "dynamic ray tracing equations" (DRT) [10].In homogeneous medium with wave speed $v$ supposed equal to the celerity denoted $c$ here, the DRT can be written as:

$$
\frac{d Q}{d s}=c . P ; \text { and } \frac{d P}{d s}=0
$$

To solve this above DRT system (2), the initial conditions must be specified at an arbitrary point $\mathrm{s}=\mathrm{s} 0$ on the central ray. We must solve this system of differential equations for $\mathrm{Q}$ and $\mathrm{P}$ with appropriate initial conditions. They must guarantee the following three conditions along the whole rays [11]: $(\operatorname{det}[Q] \neq$ $0)$, the $\left(P \times Q^{-1}\right)$ is symmetric matrix, even though $P$ and $Q$ are not symmetrical, and $\operatorname{Im}\left(P \times Q^{-1}\right)$ is a positive-definite matrix

Here, we use Hill's [12] initial data for the Green's function. The initial values for $Q$ and $P$ are respectively:

$$
Q=\frac{\omega_{r} \cdot \omega_{0}^{2}}{c} . I ; P=\frac{j}{c} . I \text { and } ; s=s_{0}
$$

In (3), $\omega_{0}$ is the initial half beam width at the frequency $f=$ $\omega_{r} / 2 \pi, I$ is the identity matrix $(2 \times 2)$. Using the initial conditions in (3), we can find the general solution of (2), and can be written as follows:

$$
Q=\left(\frac{\omega_{r} \cdot \omega_{0}^{2}}{c} .+j \cdot\left(s-s_{0}\right)\right) . I \text {; and } P=\frac{j}{c} . I
$$

In the case of homogenous media, by using (4) in (1), we return to the representation of the amplitude $u$ of the Gaussian beam in $3 \mathrm{D}$ :

$$
u\left(s, q_{1}, q_{2}\right)=\frac{c^{\frac{3}{2}}}{\omega \cdot \omega_{0}^{2}+j \cdot\left(s-s_{0}\right)} \cdot \exp \left(j \omega\left(\tau(s)+\frac{1}{2} \frac{\left(q_{1}^{2}+q_{2}^{2}\right)}{c \cdot\left(-j \frac{\omega \cdot \omega_{0}^{2}}{c} \cdot+\left(s-s_{0}\right)\right)}\right)\right)
$$

Using the geometrical configuration illustrated in Fig.1, and introducing the spherical coordination system $(r, \theta, \varphi)$, we can deduce the following factor (in 6) as function of the distance $(r)$ between the transmitter and the receiver:

$$
q_{1}^{2}+q_{2}^{2}=r \cdot \sin (\varphi) ; \text { and } s-s_{0}=r \cdot \cos (\varphi)
$$

An exhaustive discussion of the dependence of the Gaussian beam solution with beam with $\omega_{0}$ is given in [13] and [14]. The formulation of the total amplitude at the receiver is given by the integral over all Gaussian beams characterized by their takeoff angle, denoted $\varphi$, from the source is given by the following equation:

$$
u^{s f g}(M)=\int_{s} \Phi_{\varphi} \cdot u_{\varphi}\left(s, q_{1}, q_{2}, t\right) \cdot d \delta
$$

Where, is a quantity, generally complex-valued, which remains constant along the considered ray but may differ from ray to ray. It is called complex weight function. And the function $u_{\varphi}\left(s, q_{1}, q_{2}\right)$ is the Gaussian beam connected with the ray.
In (7) the domain $\delta$ is centered on the central ray, it delimits the beams propagating in the neighbor of the central ray, chosen in such way that the Gaussian beam $u_{\varphi}\left(s, q_{1}, q_{2}\right)$ outside this domain do not contribute effectively to the wave field. $\delta$ is a cone with a vertex angle $\varphi$.

$$
d \delta=\sin \varphi \cdot d \varphi \cdot d \vartheta, \quad \vartheta \in[0,2 \pi]
$$

For homogeneous medium, the ray asymptotic solution of the Helmholtz equation is given by the following equation:

$$
u(M)=\frac{1}{4 \cdot \pi \cdot r} \cdot \exp \left(j \cdot \frac{\omega}{c} \cdot r\right)
$$

Where $r$ the distance to an observation point $M$.

The GBS integral, in (7), may be evaluated asymptotically using the saddle-point method. Thus, this result must coincide with the above ray asymptotic solution in regular region. Matching both asymptotic solution of (7) and (9) we can determine the complex weight function $\Phi \varphi$. Integral (7) is evaluated by numerical quadrature with regular increment $\Delta \varphi$.

$$
u(M)=2 \cdot \pi \cdot \sum_{k=1}^{N} \Phi_{\varphi k} \cdot u_{\varphi k} \cdot \sin \left(\varphi_{k}\right) \cdot \Delta \varphi_{k}
$$

After the formulation of the scattered filed using GBS method ((7) and (10)), we analyze the influence of the main parameters of the Gaussian beam on the variation on the field amplitude. Then, we compare the solution based on Gaussian beam with the analytical solution given by (9).

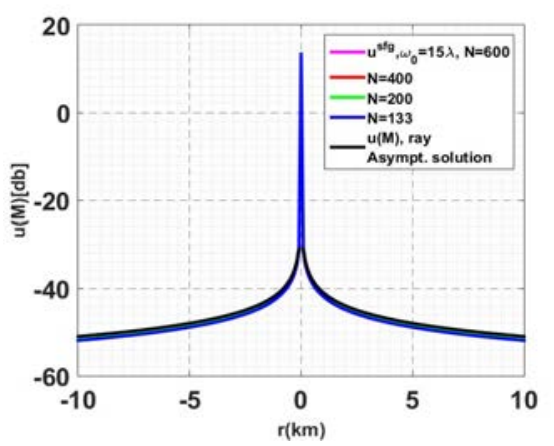

Fig. 2. Comparison between ray asymptotic solution Gaussian beam summation method for $\mathrm{N}=133,200,400,600$ the beam width is $15 \lambda$.

Fig. 2 compares the amplitude of field calculated by GBS method and ray asymptotic solution of the Helmholtz equation for a frequency equal to $10 \mathrm{GHz}$, a beam width $\left(\omega_{0}\right)$ equal to $15 \lambda$ (where $\lambda$ is the wavelength) and for different values of beams number $(\mathrm{N}): \mathrm{N}=\{133,200,400$ and 600$\}$. This simulation (Fig.2) has been realized as function of the distance ( $\mathrm{r}$ in $\mathrm{km}$ ) from the source to the receiver. Magenta, red, green, blue and lines correspond to the GBS solution for different beams number, respectively 133, 200, 400 and 600 over which the summation is down. The black line represents the ray asymptotic solution. We can observe, that the beam density in the vicinity of the central ray offers satisfactory accuracy. In fact, when the number of beam is more than 200, the GBS and the ray asymptotic are in good agreement. So, as with the usual techniques of ray tracing, a high beam density (200 for this case) is necessary for high accuracy. In Fig.3, we compare the percentage error between the ray asymptotic solution and the GBS method. We can see that for sufficient beam density, 
$\mathrm{N}=400$, the relative error remains below $4 \%$ even at $10 \mathrm{~km}$ from the source. In addition, one should note that the computations by GBS method exhibit no singularities when passing by the source point $(r=0)$, unlike the ray asymptotic solution. The proof of this result relies on the theory of systems of linear first order differential equations [15].

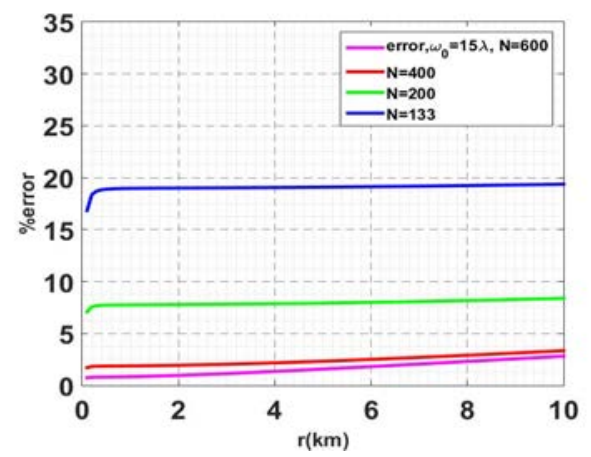

Fig. 3. Percentage error between the ray asymptotic solution and the GBS approximation of the Helmholtz's wave equation.

To estimate the RCS of a canonical target using the GBS method, we need to calculate the complex weight function. In our proposed methodologies, we can identify the complex weight function by comparing the solution established in (7) to those of physical optics (PO). PO determines the field scattered from a scatter by assuming that the field on the surface of the scatter is the geometric surface field. Since the asymptotic high-frequency treatment of superposition integral (7), should yield the same results as the standard ray method in regular regions [11], the complex weight function is determined by matching the solutions of (10) obtained by the stationary phase method and the geometrical optics solution [16]. Finally equation (10) is used for the numerical computation.

\section{NUMERICAL AND EXPERIMENTAL RESULTS: VALIDATION AND EVALUATION}

The validation of the numerical simulation results have been done in the monostatic configuration (where the transmitter and the receiver are in in the same position), which is located in an anechoic chamber $(8 \mathrm{~m} \times 5 \mathrm{~m} \times 5 \mathrm{~m})$ at ENSTA Bretagne (see Fig.4).

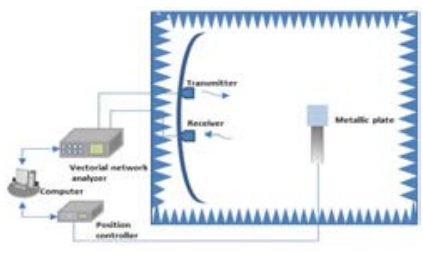

(a)

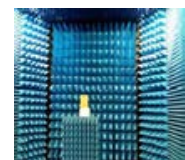

(b)

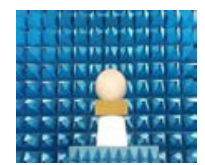

(c)
Fig. 4. (a) General description of experimental setup, (b) Target plate, (c) Radar sphere calibration.

The characteristics of various components of measurements system are:

- All walls are covered with absorbent material.

- A computer controls the Vectorial Network Analyzer (Anritsu 37347D) which operates in the frequency range from $40 \mathrm{MHz}$ to $20 \mathrm{GHz}$ and the positioning system.
- The NEWPORT positioning system with an angular resolution equal to $0.01^{\circ}$ and an angle vary between $-90^{\circ}$ and $90^{\circ}$.

- An elevation motor for adjusting the height of the target.

In Fig.5, we show the RCS variation of a rectangular flat plate of sides $(1 \mathrm{~m} \times 1 \mathrm{~m})$.

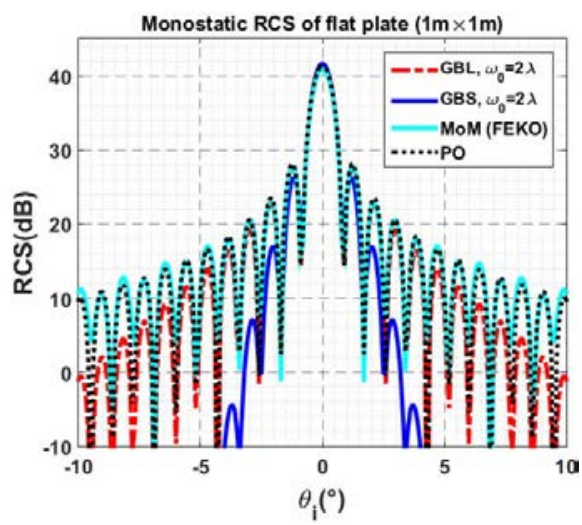

Fig. 5. Monostatic RCS of a flat plate, in $v v$ polrization, computed using GBL method, GBS,PO and MoM, for beam width $\omega_{0}=2 \lambda$.

In this simulation (Fig.5), we set the azimuth angles $\varphi_{i}$ to zero; the incident angle $\theta_{\mathrm{i}}$ varies from $-10^{\circ}$ to $10^{\circ}$ and the other acquisition parameters are: $\mathrm{f}=10 \mathrm{GHz}$, the beam width $\omega_{0}=2 \lambda$, beams number $\mathrm{N}=200$. The GBS are compared with its variety approach GBL (Gaussian Beam Launching), the asymptotic PO model, and the rigorous MoM method (in FEKO). Comparing the curve of RCS using GBS is in blue line, GBL technique in bred line with the other models; we observe that they match rigorously the PO solution and MoM for the main beam. In fact, PO and MoM accurately models the main beam, and the modeled peak width and location match GBS and GBL methods. GBS and GBL accurately model the main beam, without mitigation, the diagrams are also consistent, and the modeled peak width and location match the PO solution.

From the simulation results (in Fig.5) we can conclude that the GBS method model very well the variation of the scattered field in the specular direction. The deviation outside specular direction can be considered through broadening the range of validity of the GBS by taking into account the edge diffraction.

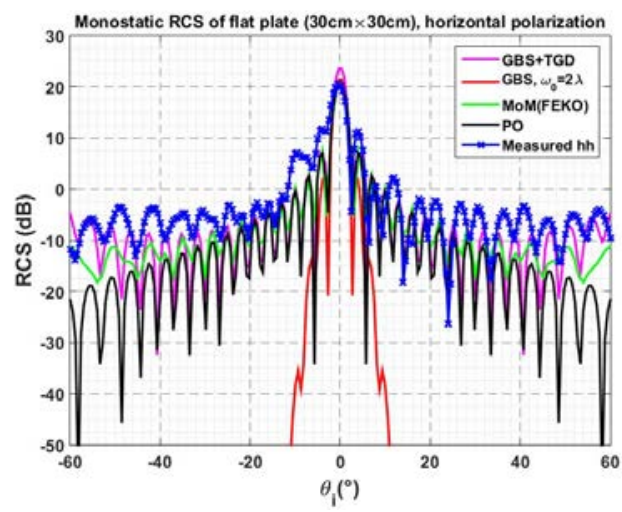

Fig. 6. Compraison between GBS, the numerical models and the experimental measurements in $h h$ polrarization: $(30 \mathrm{~cm} \times 30 \mathrm{~cm})$. 


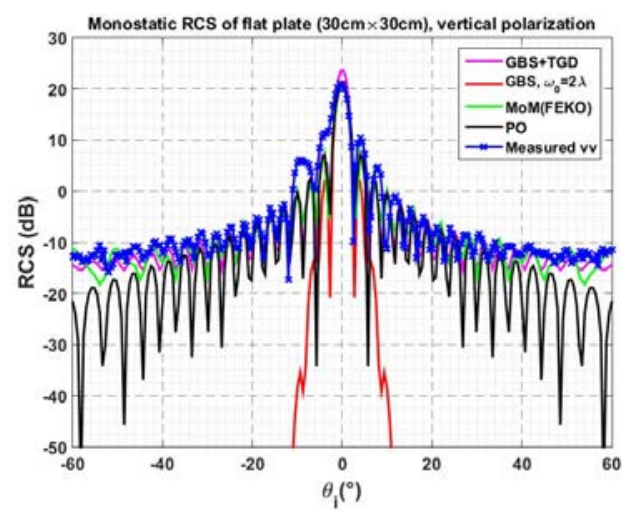

Fig. 7. Compraison between GBS, the numerical models and the experimental measurements in $v v$ polrarization: $(30 \mathrm{~cm} \times 30 \mathrm{~cm})$.

To consider the edge diffraction contribution, we need to use the method of the Geometric Theory of Diffraction (GTD) [17]. In fact, the diffraction field when incident field strikes the edges is calculated from the GTD and is accounted in the complex weighing function in the integral (7). In Fig.6, and Fig.7 we compare the GBS method with experimental measurements. For a beam width of $\omega_{0}=2 \lambda$, a beam number equal to 200 , an incident angle $\theta \mathrm{i}$ varies from $-60^{\circ}$ to $60^{\circ}$ and a frequency of $10 \mathrm{GHz}$, the size of flat plate is $(10 \lambda \times 10 \lambda)$.

The comparison between GBS, with accounting and without accounting the edge diffraction contribution, is shown. Comparison is also performed with PO and MoM. From this comparison results we can see that when the diffraction is accounted we obtain values of the RCS which get closer to those given by the experimental measurements and the rigorous MoM method. In addition, the experimental RCS measurements show non-symmetry between the angle of $-10^{\circ}$ to $0^{\circ}$ and $0^{\circ}$ to $10^{\circ}$. This is due to the separation between the transmitting and receiving antennas which equal $2^{\circ}$ (quasimonostatic configuration).

The experimental validation results shows that GBS give a higher accurate representation of the scattered field and offer very interesting perspectives for complex targets such as cavity and corner for example (see Fig.8).

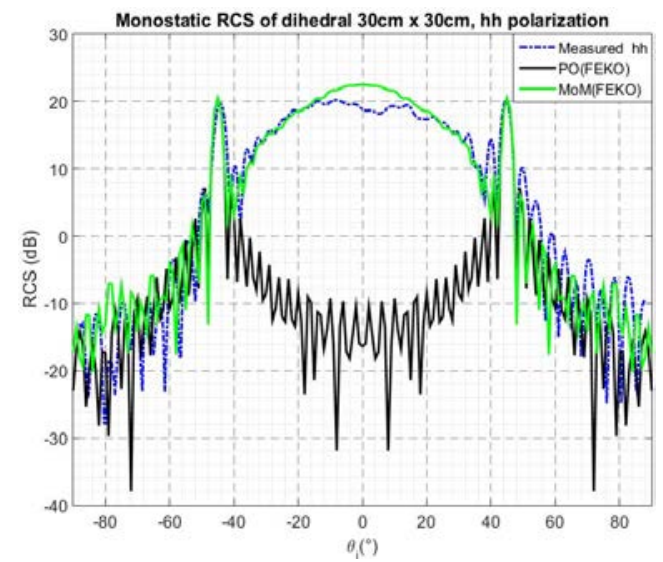

Fig. 8. RCS of rectangular dihedral corner reflector ( $\mathrm{f}=10 \mathrm{GHz}$ ) Experimental and numerical models.

\section{CONCLUSION AND FUTURE WORK}

In the present paper, we have applied a new technique in the electromagnetic scattering from a radar targets. In the GBS technique, the total field at the receiver is represented by the integral over all Gaussian beams propagating in the neighbor of the receiver. We have carried out the theoretical formulation of the GBS technique and a study of influence parameters (beams number and beams width). Then, we have introduced a numerical simulation of the electromagnetic scattering by a flat plate using GBS method. In addition, we have presented the experimental measurements of RCS of canonical and complex targets. The results of RCS using GBS method were compared and validated by the experimental measurements. The study of the RCS of different complex objects (such as dihedral and trihedral corner reflectors,...) using GBS method is one of the perspectives of the work presented in this paper

\section{REFERENCES}

[1] F.Weinmann, "Ray tracing with PO/PTD for RCS modeling of large complex objects," IEEE Trans. Antennas and Propagation, vol. 54, no. 6, pp. 1797-1806, Jun. 2006.

[2] R. Harrington, "Field computation by moment methods," Wiley-IEEE Press, 1993.

[3] P.O. Leye, A. Khenchaf, and P. Pouliguen, "The Gaussian Beam Summation and the Gaussian Launching Methods in Scattering Problem," J. Elect. Analysis and Applications, vol. 8, pp.219-225, 2016.

[4] M. Katsav and E. Heyman, "Gaussian Beam Summation Representation of Beam Diffraction by an Impedance Wedge: A 3D Electromagnetic Formulation Within the Physical Optics Approximation," IEEE Trans. Antennas Propagation, vol. 60, no. 12, pp. 5843-5858, 2012.

[5] V. Červený, "Summation of paraxial Gaussian beams and of paraxial ray approximations in inhomogeneous anisotropic layered structures," In Seismic waves in Complex 3-D Structures, no. 10, pp. 121-159, 2000.

[6] M. M. Popov, "A new method of computation of wave fields using Gaussian beams," Wave Motion., vol. 4, pp. 85-97, 1982.

[7] H. T. Chou and P. H. Pathak, "Uniform asymptotic solution for electromagnetic reflection and diffraction of an arbitrary Gaussian beam by a smooth surface with an edge," Radio Science, vol. 32, no.14, pp. 1319-1336, 1997.

[8] V. Červený, "Expansion of a Plane Wave into Gaussian Beams," Stidia geoph. And geod., vol. 26, pp. 120 - 131, 1982.

[9] V. Červený, "Seismic Ray Theory," Cambridge: Cambridge University Press, 2001.

[10] M. M. Popov, "New method of computation of wave fields in high frequency approximation", Mathematical problems in the theory of wave propagation. J. Soviet Math., 20:1 ,1869-1882, 1982.

[11] V. Červený, "Gaussian beam synthetic seismograms," Journal of Geophysics, vol.58, pp. 44-72, 1985.

[12] N. R. Hill, "Gaussian beam migration," Geophysics, vol. 55, no.11, pp. 1416-1428, 1990.

[13] V. Červený, «Computation of wave field in homogeneous media,» Geophys. J. R. astr. Soc., vol. 70, pp. 109-128, 1982.

[14] B. S. White, A. Norris, A. Bayliss and R. Burridge, "Some remarks on the Gaussian beam summation method," Geophysical Journal of the Royal Astronomical Society, vol. 89, pp. 579-636, 1987.

[15] B. Bleistein, "Mathematics of Modeling, Migration and Inversion with Gaussian Beams," Colorado, USA, 2008.

[16] H. T. Chou, P. Pathak et R. J. Burkholder, "Novel Gaussian Beam Method for the Rapid Analysis of Large Reflector Antennas," IEEE Trans Antennas and Propagation, vol. 49, n%16, pp. 880-893, 2001.

[17] J. B. Keller, "Geometrical Theory of Diffraction," Journal of Optical Society of America, vol. 52, pp. 116 - 130, 1962. 